\title{
Analysis of the Working Cable System of Single-span Circulating Ropeway
}

\author{
Jian Qin ${ }^{1}$, Liang Qiao ${ }^{1}$, Jun Chen ${ }^{1}$, Jiancheng Wan ${ }^{1}$, Ming Jiang ${ }^{1}$ and Chunhua $\mathrm{Hu}^{1}$ \\ ${ }^{1}$ China Electric Power Research Institute, 100055, Beijing, China
}

\begin{abstract}
Based on the analysis of the mutual interaction between bearing cable and traction cable of single-span circulating ropeway, the calculation method is proposed for working cables of ropeway with arbitrary loads. The 5 sets nonlinear equations are set up for the working cables according to the equal spans and altitude differences of the bearing cable and the traction cable, the constant of the load running space and the bearing cable length, and the tension balance of bearing cable and traction cable beside load. The resolving method is proposed based on the Newton iterative method. The correctness and feasibility of the method are proved by the results of engineering test. From the solution, the tension and coordinate of working cables can be obtained accurately, which is meaningful for the design and analysis of the freight ropeway.
\end{abstract}

\section{Single-span freight ropeway}

Aerial freight ropeway has strong adaptability for natural terrain and perfect climbing ability, so it can easy across mountains and rivers. It is widely used in forestry, construction, mine and other industries [1-3].

For the single-span ropeway, the accurate calculation of bearing cable tension is the key of the design and selection in ropeway system [4]. In the past, only the influence of weights of cable and loads are considered during the tension calculation of bearing cable [5-6], and the mutual interaction between bearing cable and traction cable is always ignored which will make the big error in tension calculation of the bearing cable. In this paper, a calculation method is proposed of mutual interaction between the bearing cable and the traction cable based on the elastic catenary theory.

\section{Ropeway with arbitrary loads}

The ropeway mainly includes working cables, brackets, trolleys and tractor. Working cables includes bearing cable and traction cable. The bearing cable is the important part of ropeway, which bears the loads of trolleys and cargos. The traction cable is a closed rope and driven by the tractor to move along the bearing cable in the ropeway [7].

(1) Parameters of ropeway

The single-span circulating ropeway is shown in Figure 1 . The span between brackets is $L_{s}$, and altitude difference is $h_{s}$.

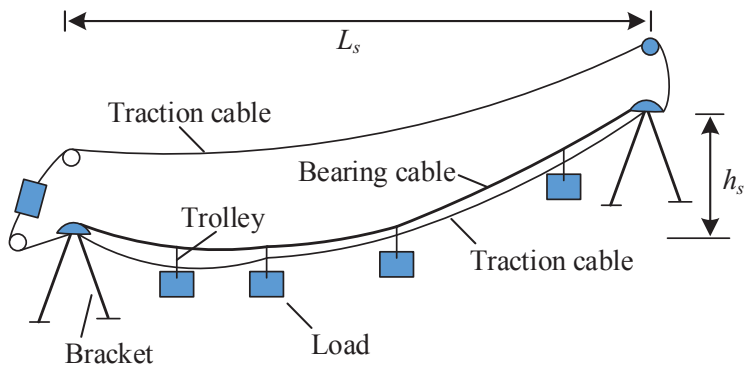

Figure 1. The single-span circulating ropeway

The length of bearing cable $s_{0}^{l}$ and length of traction cable $s_{0}^{t}$ can be measured after the erection of ropeway.

(2) Parameters of loads

There are $m$ loads on the ropeway with the weights $G_{i}(i=1, \ldots, m)$ respectively. The lengths of traction cable between loads are $s_{i}^{G}(i=1, \ldots, m-1)$, which are kept constant during movement.

The length of traction cable from the tractor to the first load is defined as $s_{0}^{G}$ and the length between the last load and tractor is defined as $s_{m}^{G}$, shown as Figure 2 . 


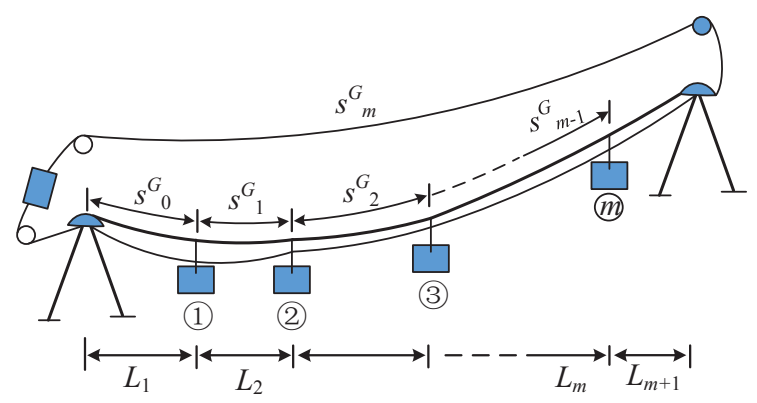

Figure 2. The single-span circulating ropeway

The working cables are divided by centralized loads as several catenary sections which only bear the self-weight of the cable. The beginning of every section is $A$, the ending is $B$. So there are $m+1$ bearing cable sections, and $m+2$ traction cable sections.

(3) Formulas of section

Assume the initial length of every section cable is $s_{i}$, horizontal tension is $H_{i}$, vertical tension of $A$ is $V_{A i}(i=1, \ldots, m+1)$, the vertical tension of $B$ can be expressed as:

Here $q$ is the unit length weight of cable.

$$
V_{B i}=V_{A i}+q s_{i} \quad(i=1, \ldots, m+1)
$$

The spans and altitude differences of every section are[7]:

$$
\begin{aligned}
& L_{i}=\frac{H_{i}}{q}\left[\frac{q s_{i}}{E A_{0}}+\operatorname{asinh} \frac{V_{B i}}{H_{i}}-\operatorname{asinh} \frac{V_{A i}}{H_{i}}\right] \\
& h_{i}=\frac{V_{B i}^{2}-V_{A i}^{2}}{2 q E A_{0}}+\frac{H_{i}}{q}\left(\sqrt{1+\frac{V_{B i}^{2}}{H_{i}^{2}}}-\sqrt{1+\frac{V_{A i}^{2}}{H_{i}^{2}}}\right)^{(i=1, \ldots, m+1)}
\end{aligned}
$$

Here $E A_{0}$ is the product of section area and elasticity modulus.

These variables above can represent bearing cable or traction with the superscripts $l$ or $t$ respectively.

\section{Analysis of bearing cable and traction cable}

The mutual interaction between bearing cable and traction cable of single-span ropeway is mainly reflected from the following aspects:

(1) The equal span and altitude difference of bearing cable and traction cable

As shown in Figure 2, the points A and B coincide in every section of bearing cable and traction cable that means the span and altitude difference of every section are equal. So the $2(m+1)$ equations below are obtained:

(2) Constant of the total span and altitude difference

$$
\begin{aligned}
& L_{i}^{l}-L_{i}^{t}=0 \\
& h_{i}^{l}-h_{i}^{t}=0
\end{aligned}(i=1, \ldots, m+1)
$$

No matter where the loads move in single-span ropeway, the sum of section spans is equal to whole horizontal span and the sum of section altitude difference is equal to whole altitude difference.

For return traction cable, the equations are obtained as:

$$
\begin{aligned}
& \sum_{j=1}^{m+1} L_{j}^{l}-L_{s}=0 \\
& \sum_{j=1}^{m+1} h_{j}^{l}-h_{s}=0
\end{aligned}
$$

(3) Tension balance of bearing cable and traction cable beside load

$$
\begin{aligned}
& L_{m+2}^{t}-L_{s}=0 \\
& h_{m+2}^{t}-h_{s}=0
\end{aligned}
$$

The bearing cable and traction cable are connected by the loads, shown as Figure 3(a). The number of cables beside load is $i$, $i+1$ respectively.

The wheel of trolley rolls on the bearing cable and makes cable bent down. Therefore, the tangential tensions of bearing cable beside load are equal, shown as Figure 3(b). So there are $m$ equations of tension balance:

$$
T_{B i}^{l}-T_{A i+1}^{l}=0(i=1, \ldots, m)
$$




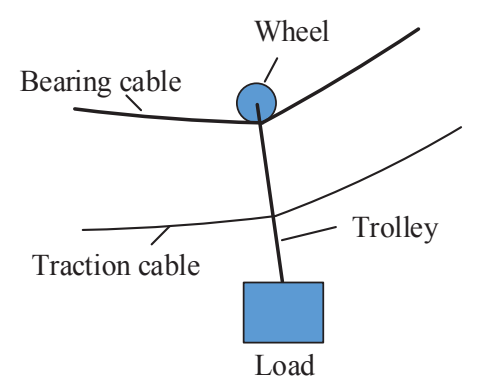

(a) Connect of bearing cable of traction cable

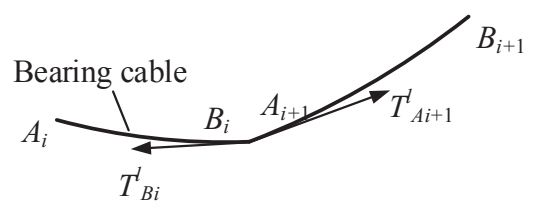

(b) Tangential tension of bearing cable

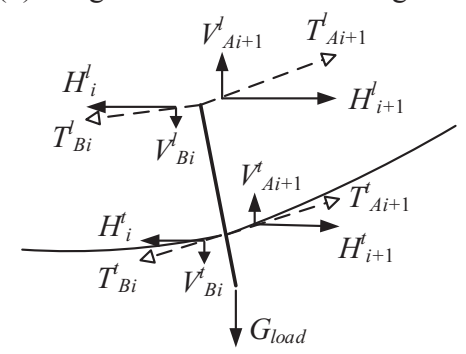

(c) Force analysis of trolley

Figure 3. Force analysis of bearing cable and traction cable beside load

The Force analysis of trolley is shown in Figure 3(c), the $2 m$ tension balance equations in the horizontal and vertical directions can be obtained:

(4) Space constant of loads

$$
\begin{aligned}
& H_{i+1}^{l}-H_{i}^{l}+H_{i+1}^{t}-H_{i}^{t}=0 \\
& V_{A i+1}^{l}-V_{B i}^{l}+V_{A i+1}^{t}-V_{B i}^{t}-G_{i}=0
\end{aligned}(i=1, \ldots, m)
$$

The space between loads pulled by traction cable is unchanged during the movement. So we have $m+1$ equations.

$$
\sum_{j=g_{i}+1}^{g_{i+1}} s_{j}^{t}-s_{i}^{G}=0(i=0, \ldots, m-1)
$$

and

$$
\sum_{j=g_{m}+1}^{2+m} s_{j}^{t}-s_{m}^{G}=0
$$

(5) Length constant of bearing cable

For single-span ropeway, the length of bearing cable has remained unchanged.

$$
\sum_{j=1}^{m+1} s_{j}^{l}-s_{0}^{l}=0
$$

\section{Solution of the nonlinear equations of working cable}

Taking the section length $s_{i}^{l}, s_{i}^{t}$, horizontal tension $H_{i}^{l}, H_{i}^{t}$, vertical tension $V_{A i}^{l}, V_{A i}^{t}(i=1, \ldots, m+1)$ of the bearing cable and traction cable as the unknowns, the nonlinear equation set of single-span ropeway can be established based on interaction relationship of the working cable.

The number of unknown variables is $6 m+9$ and equal to the nonlinear equations number. Therefore, the nonlinear equations are closed, which can be expressed as:

$$
F(X)=0
$$

In which $X=\left[s_{1}^{l}, \ldots, s_{m+1}^{l}, H_{1}^{l}, \ldots, H_{m+1}^{l}, V_{1}^{l}, \ldots, V_{m+1}^{l}, s_{1}^{t}, \ldots, s_{m+2}^{t}, H_{1}^{t}, \ldots, H_{m+2}^{t}, V_{1}^{t}, \ldots, V_{m+2}^{t}\right]^{T}$.

Newton iterative method can be used to solve the nonlinear equation set. Considered that Newton method is sensitive to the initial value, the parabolic analytical solutions is used as the initial iteration value.

(1)The length $s_{i}^{t}(i=1, \ldots, 2+m)$ of every section divided by load point can be obtained according to the whole traction cable length and load distribution. Every section length of bearing cable is calculated in proportion.

$$
s_{i}^{l 0}=\frac{s_{0}^{l}}{s_{0}^{t}} s_{i}^{t} \quad(i=1, \ldots, 1+m)
$$

(2)According to bearing cable length $s_{0}^{l}$, the sag can be calculated basis of parabolic equation:

$$
f=\sqrt{\frac{3\left(s_{0}^{l}-L_{s}\right)}{8 L_{s}}}
$$

And then the horizontal tension of every section is obtained:

$$
H^{l}=\frac{q^{l} L_{s}^{2}}{8 f}
$$

It is known from the balance conditions that the initial horizontal tension of every section is same.

$$
H_{1}^{l 0}=H_{2}^{l 0}=\cdots=H_{m+1}^{l 0}=H^{l}
$$


(3)The initial vertical tension of point $A$ of first section can be obtained basis of sag and span.

$$
V_{1}^{l 0}=\frac{4 f+h_{s}}{L_{s}}
$$

Other sections' vertical tension can be calculated refer to equation below:

$$
V_{i+1}^{l 0}=V_{i}^{l 0}+q^{l} s_{i}^{l 0}+G_{i} \quad(i=1, \ldots, m)
$$

So the initial iteration value of bearing cable is:

$$
X^{l 0}=\left[s^{10}{ }_{1}, s^{l 0}{ }_{2}, \ldots, s^{l 0}{ }_{1+m}, H^{l 0}{ }_{1}, H^{l 0}{ }_{2}, \ldots, H^{l 0}{ }_{1+m}, V^{l 0}{ }_{1}, \ldots, V^{l 0}{ }_{1+m}\right] .
$$

And the initial iteration value of traction cable can be obtained in the same way:

The initial iteration value of the nonlinear equations is:

$$
X^{t 0}=\left[s^{t 0}{ }_{1}, S^{t 0}, \ldots, s^{t 0}{ }_{1+m}, H^{t 0}{ }_{1}, H^{t 0}{ }_{2}, \ldots, H^{t 0}{ }_{1+m}, V^{t 0}{ }_{1}, \ldots, V^{t 0}{ }_{1+m}\right] .
$$

$$
X^{0}=\left[X^{t 0}, X^{t 0}\right]^{T} .
$$

After $X$ is calculated, the vertical tension $V_{x}$, tangential tension $T_{x}$ and the horizontal and vertical coordinates of any point of the working rope at length $s_{x}$ can be obtained respectively.

\section{Example}

In the single-span cableway experiment, $L_{s}=218.18 \mathrm{~m}, h_{s}=118.66 \mathrm{~m}, E A_{0}^{l}=25 \mathrm{MN}, q^{l}=1.6 \mathrm{~kg} / \mathrm{m}, E A_{0}^{t}=9 \mathrm{MN}, q^{t}=0.8 \mathrm{~kg} / \mathrm{m}$. The length of bearing cable is $249.12 \mathrm{~m}$.

The first load case is $G_{1}=850 \mathrm{~kg}$, The $2^{\text {nd }}$ case is $G_{1}=850 \mathrm{~kg} G_{2}=425 \mathrm{~kg}$, and $s_{1}^{G}=21 \mathrm{~m}$.

In the case 1 , the load moves from the $11 \mathrm{~m}$ to $87 \mathrm{~m}$. The measured and calculated tangential tension of initial point with the load position is shown in Figure 4(a). In the case 2, the load moves from the $11 \mathrm{~m}$ to $83 \mathrm{~m}$. The relation is shown in Figure 4(b).

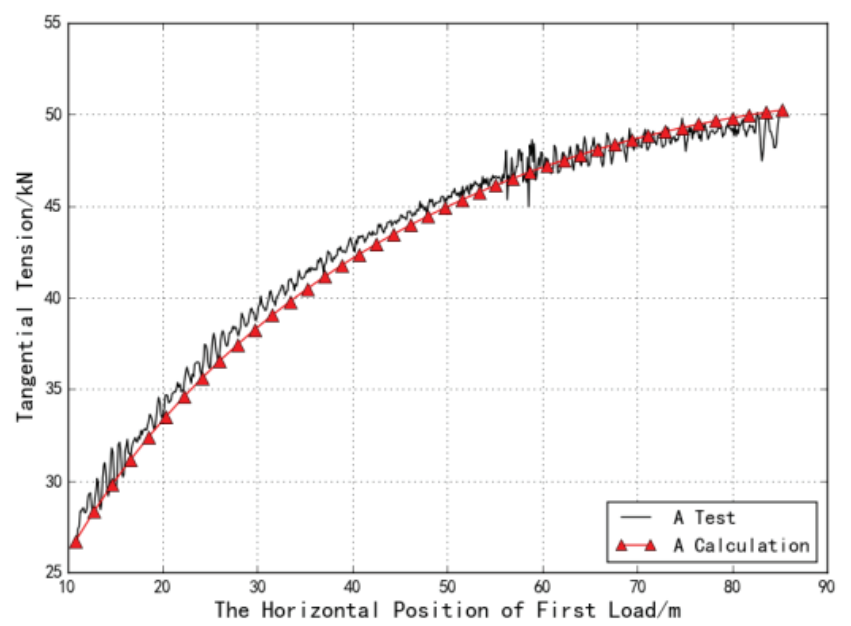

(a) Result of case 1

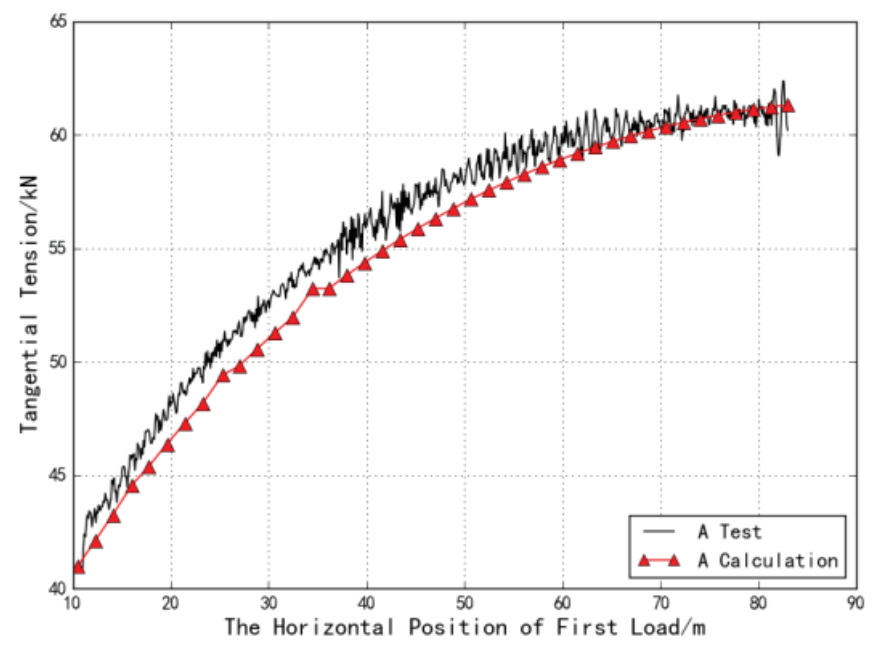

(b) Result of case 2

Figure 4. The results of the tangential tension with the load position in case 1 and case 2

From the figure 4, the curve of calculated tangential tension is consistent with the experimental values, and the maximum error is only $0.16 \%$ in case $1,1.70 \%$ in case 2 .

Therefore, the accuracy of method is verified which can meet the requirements of engineering applications. 


\section{Conclusions}

In this paper, the nonlinear equation set of working cable of single-span circulating ropeway is proposed. The set includes the constant of spans and altitude differences between bracket, the tension constant of the bearing cable and the traction cable beside load, the length constant of the load running space and the bearing cable length.

The calculation method is fast and good in convergence. It is an effective solution for interaction problem of bearing cable and traction cable, which can meet with requirement of the circulating ropeway design.

\section{Acknowledgment}

This research was supported by Science and Technology Research Project of State Grid (Research on the calculation method of multi-bearing cable ropeway and the application in UHVDC).

\section{References}

1 Zhao Yueyu, Wang Lianhua, etc. Direct Treatment and Discretization of Non-linear Dynamics of Suspension Cable[J]. Acta Mechanica Sinica. 2005, 37(3):329-338.

2 Cheng Daye. Exact Element Method for Analysis of Cable Structures[D]. Beijing: Tsinghua University, 2005.

3 Li Bin, Li Yinghui. Dynamic Modeling and Simulation of Flexible Cable with Large Sag[J]. Applied Mathematics and Mechanics. 2000, 21(6): 640-646.

4 Cheng, S. P. , Perking, N. C. Free Vibration of A Sagged Cable Supporting A Discrete Mass[J]. Journal of the Acoustical Society of America. 1992a, 91(5): 2654-2662.

5. Gavrilov, S.. Non-stationary problems in dynamics of a string on an elastic foundation subjected to a moving load[J]. Journal of Sound and Vibration, 1999, 222(1), 345-361.

6. Di Tomasso, F., Di Egidio, A., Luongo, A., 2003. Modelling and Dynamic Analysis of Strings Carrying A Single Mass. In: Proc. of 16th AIMETA Congress, CD-Rom, Ferrara, Italy (in Italian).

7. Qin Jian, Xia Yongjun. The Matrix Iteration Method for Analysis of Suspension Cable Based on Segmental Catenary Theory [J]. Chinese Journal of Engineering Design, 2013, 20(5): 404 408. 